\title{
Vitamin D3 promotes autophagy in THP-1 cells infected with Mycobacterium tuberculosis
}

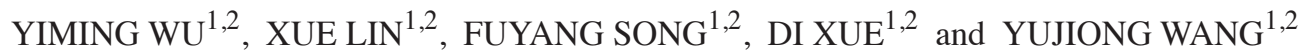 \\ ${ }^{1}$ Key Laboratory of The Ministry of Education for Conservation and Utilization of Special Biological Resources \\ in The West; ${ }^{2}$ College of Life Science, Ningxia University, Yinchuan, Ningxia 750021, P.R. China
}

Received September 20, 2021; Accepted December 16, 2021

DOI: $10.3892 /$ etm.2022.11165

\begin{abstract}
Tuberculosis (TB) is a major disease that causes mortality worldwide. The lethality of this disease is a result of the contagious bacteria Mycobacterium tuberculosis (M.tb). Infection can inhibit phagosomal maturation, with $M . t b$ mainly attacking macrophages and inhibiting autophagy and apoptosis. Vitamin D has been used to treat tuberculosis, whereby the active metabolite, 1,25-dihydroxyvitamin D, may enhance the immune response to $M$.tb. Moreover, macrophages infected with $M . t b$ have a high demand for $\mathrm{Ca}^{2+}$. However, the mechanisms by which vitamin D3 protects against and treats TB remain unclear. In the present study, MTT assay showed that vitamin D3 decreased the viability of THP-1 cells in a dose- and time-dependent manner. Autophagy-related factors in THP-1 cells infected with M.tb were analyzed by western blotting and RT-qPCR and the results demonstrated that vitamin D3 significantly increased the expression level of p62, LC3II/LC3I, Beclin-1, ATG-5 and AMPK in THP-1 cells following M.tb infection. The $\mathrm{Ca}^{2+}$ concentration assay demonstrated that vitamin D3 may promoted cellular autophagy by inhibiting the concentration of $\mathrm{Ca}^{2+}$. Furthermore, the effect of vitamin D3 on M.tb infection was also assessed using Balb/c mice; pulmonary injury was assessed by $\mathrm{H} \& \mathrm{E}$ staining of the lungs tissue. The results demonstrated that vitamin D3 markedly attenuated cellular damage caused by M.tb infection. In conclusion, the present study indicated that vitamin D3 may activate cell autophagy signals by inhibiting the concentration of $\mathrm{Ca}^{2+}$. These data may improve understanding of the effect of vitamin D3 on M.tb infection and help determine the underlying mechanism of vitamin D3 to alleviate and treat the inflammatory response caused by TB.
\end{abstract}

Correspondence to: Dr Yujiong Wang or Dr Di Xue, College of Life Science, Ningxia University, 539, Helanshan West Road, Yinchuan, Ningxia 750021, P.R. China

E-mail: wyj@nxu.edu.cn

E-mail: xue_di@yeah.net

Key words: Mycobacterium tuberculosis, vitamin D3, $\mathrm{Ca}^{2+}$, autophagy

\section{Introduction}

Tuberculosis (TB) caused by Mycobacterium tuberculosis (M.tb) remains the principal killer among global infectious diseases (1). According to a WHO report 2018, it is estimated that 10 million persons had incident TB, and 1.5 million TB-related mortalities occurred (2). M.tb is unique among bacterial pathogens as it can survive in the body for several years. Therefore, finding effective therapeutic strategies to manage this infectious disease is important (3). M.tb weakens host immune function and therefore enables its long-term coexistence with the host (4). M.tb virulence factors exhibit numerous modes of action, including preventing the formation and acidification of phagolysosomes, inhibiting the formation of autophagosomes, resisting oxidative stress and inhibiting cell autophagy (5). Moreover, $\mathrm{Ca}^{2+}$ is a crucial secondary messenger in cells that regulates cell cycle progression, proliferation and autophagy (6). Its role in cell proliferation and autophagy implies that increased or weakened $\mathrm{Ca}^{2+}$ signals may result in cell death (7).

A previous study demonstrated that vitamin D3 induces autophagy in numerous types of cancer cells, such as lung cancer and prostate cancer (8), which suggests that the induction of autophagy may be a mechanism whereby vitamin D3 exerts an anti-cancer effect (9). Another study reported that vitamin D3 can regulate the immune response to M.tb, which indicated that vitamin D3 serves a vital role in TB treatment (10). Furthermore, vitamin D3 serves an extensive role in $\mathrm{Ca}^{2+}$ homeostasis (11). Intracellular $\mathrm{Ca}^{2+}$ is also an essential secondary messengers in the regulation of autophagy (12). However, the role of vitamin D3 in regulating cell autophagy and immunity is still poorly understood.

Macrophages are a vital component of the immune system and serve numerous roles in the immune response, such as phagocytosis, bactericidal and in the secretion of inflammatory factors (interleukin IL-1 $\beta$, tumor necrosis factor $\alpha$ and IL-6) (13). The THP-1 human leukemia monocyte cell line has been widely used as a model to study the immune response of monocytes (14). In the present study, the effect of vitamin D3 on THP-1 cells infected by M.tb was explored, including its function in the modulation of intracellular $\mathrm{Ca}^{2+}$ and the induction of autophagy. THP-1 cells were incubated with $M . t b$ and the autophagic process and $\mathrm{Ca}^{2+}$ concentration of the host cells following vitamin D3 treatment were investigated. Animal 
experiments were used to further verify the role of vitamin D3 in promoting autophagy after M.tb infection.

\section{Materials and methods}

Animals. A total of 16 male Balb/c mice (age, 6-8 weeks; weight, $20 \pm 5 \mathrm{~g}$ ) were obtained from the Jackson Laboratory. All animal experiments and experimental protocols were approved by the Ethics Committee of Ningxia University (Yinchuan, China; approval no. 2020-013). The mice were maintained in a pathogen-free facility at a constant temperature $\left(24 \pm 1^{\circ} \mathrm{C}\right)$ and $50-55 \%$ humidity with a 12 -h light/dark cycle and were allowed to eat and drink ad libitum. M.tb purchased from Chinese Center for Disease Control and Prevention was grown in Middlebrook $7 \mathrm{H} 9$ broth (BD Diagnostic; Becton, Dickinson and Company) supplemented with $10 \%$ OADC and $0.05 \%$ Tween -80 at $37^{\circ} \mathrm{C}$ in a tissue incubator with an atmosphere of $5 \% \mathrm{CO}_{2}$. After one week of acclimatization, the mice were randomly divided into the following four treatment groups ( $\mathrm{n}=4$ mice/group): i) Control group (50 $\mu 10.9 \%$ normal saline administered intranasally); ii) M.tb group [50 $\mu 1 M . t b(50 \mu \mathrm{g} / \mathrm{ml})$ administered intranasally]; iii) vitamin D3 group [50 $\mu 1$ vitamin D3 (Beyotime Institute of Biotechnology; $5 \mu \mathrm{mol} / \mathrm{ml}$ ) administered intranasally]; and iv) vitamin D3 + M.tb group [50 $\mu 1$ vitamin D3 $(5 \mu \mathrm{mol} / \mathrm{ml})$ and $50 \mu \mathrm{l} M . t b(50 \mu \mathrm{g} / \mathrm{ml})$ administered intranasally]. Intranasal administration was performed under $1.5-2 \%$ isoflurane anesthesia with $50 \mu \mathrm{l}$ of the saline, $M . t b$ and vitamin D3 (Beyotime, China) instilled into one nostril. Treatment was repeated once a day for seven days in each treatment group. The animals were placed into the induction box (1.5-2\% isoflurane). Once the animals were completely anesthetized (3-5 min; animals did not attempt resume prone position), anesthesia was maintained using $0.5-1.0 \%$ isoflurane.

Histological examination. Mice were sacrificed by cervical dislocation at indicated time points (7 days after M.tb and vitamin D3 treatment). Subsequently, the lungs were extracted from the mice, washed once with PBS and fixed in 4\% paraformaldehyde for $24 \mathrm{~h}$ at room temperature. the fixed tissue was embedded in paraffin and sliced into $4-\mu$ m-thick sections using a microtome. Samples were then stained with H\&E for $10 \mathrm{~min}$ at room temperature and images captured with a light microscope (magnification, x100).

Cell culture. The monocytic THP-1 cell line (ATCC) was cultured in RPMI 1640 medium (Gibco; Thermo Fisher Scientific, Inc.) supplemented with $10 \%$ FBS (Thermo Fisher Scientific, Inc.) in a $5 \% \mathrm{CO}_{2}$ incubator at $37^{\circ} \mathrm{C}$. Before incubation, the cells were seeded into a 6 -well plate at a cell density of $2 \times 10^{6}$ cell/well and treated with $100 \mathrm{ng} / \mathrm{ml}$ phorbol 12 -myristate 13 -acetate (PMA) at $37^{\circ} \mathrm{C}$ for $24 \mathrm{~h}$ to transform into adherent macrophages. A vision-based automatic cell counter was used to determine the total number of cells.

Assessment of cell viability. The MTT assay (Thermo Fisher Scientific, Inc.) was used to assess cell viability. THP-1 cells were seeded into a 96 -well plate at a density of $5 \times 10^{3}$ cells/well and treated with $100 \mathrm{ng} / \mathrm{ml}$ phorbol 12-myristate 13-acetate
(PMA) at $37^{\circ} \mathrm{C}$ for $24 \mathrm{~h}$ to transform into adherent macrophages. Subsequently, the resulting THP-1 cells was treated with different concentrations of vitamin D3 $(0,5,20,50$ and $100 \mu \mathrm{mol} / \mathrm{ml}$ ) at $37^{\circ} \mathrm{C}$ for $24 \mathrm{~h}$ or treated with vitamin D3 $(5 \mu \mathrm{mol} / \mathrm{ml})$ at $37^{\circ} \mathrm{C}$ for $0,6,12,24$ and $36 \mathrm{~h}$. Following incubation, $20 \mu 1$ MTT solution was added to each well, according to the manufacturer's instructions. The plate was then transferred to an incubator for another $4 \mathrm{~h}$ at $37^{\circ} \mathrm{C}$; DMSO solution $(100 \mu \mathrm{l})$ was subsequently added to each well to dissolve the purple formazan. Finally, the absorbance $(560 \mathrm{~nm})$ was measured using a microplate reader (Bio-Rad Laboratories, Inc.).

$\mathrm{Ca}^{2+}$ concentration assay. THP- 1 cells were seeded at a density of $5 \times 10^{3}$ cells/well in 96-well plates and treated with $100 \mathrm{ng} / \mathrm{ml}$ PMA at $37^{\circ} \mathrm{C}$ for $24 \mathrm{~h}$ to transform into adherent macrophages. Then the cells of different treatment groups were incubated with M.tb (MOI:35) and vitamin D3 (5, 10 and $20 \mu \mathrm{mol} / \mathrm{ml})$ at $37^{\circ} \mathrm{C}$ for $24 \mathrm{~h}$. Cytosolic $\mathrm{Ca}^{2+}$ concentration was quantified using Fluo-4 acetoxymethyl (AM; Thermo Fisher Scientific, Inc.). In brief, Fluo-4 AM was diluted in PBS and the cells were incubated at a final concentration of $1 \mu \mathrm{M}$ for $30 \mathrm{~min}$ at $37^{\circ} \mathrm{C}$ to ensure that Fluo-4 AM was fully converted into Fluo-4 intracellularly. Cells were then washed three times with PBS solution, and the fluorescence intensity was assessed by acquiring emissions at $494 \mathrm{~nm}$ using flow cytometry. (Thermo Fisher Scientific, Inc.). FlowJo version 10 software (FlowJo, LLC) was used for flow analysis. The result was compared with the control group.

Western blotting. M-PER Mammalian Protein extraction reagent (Thermo Fisher Scientific, Inc.) was used to extract total protein from cells in the different treatment groups. A BCA kit was used to determine protein concentration. Total protein (10 $\mu \mathrm{g}$ per lane) was mixed with $6 \mathrm{X}$ loading buffer and separated using SDS-PAGE on a $10 \%$ gel; separated proteins were subsequently transferred to a PVDF membrane. After blocking with Superblock Blocking Buffer (Thermo Fisher Scientific, Inc.) at $37^{\circ} \mathrm{C}$ for $1 \mathrm{~h}$, the membrane was incubated with the following primary antibodies: anti-LC3 (cat. no. 14600-1-AP; 1:1,000; ProteinTech Group, Inc.), anti-sequestosome-1 (p62, cat. no. 18420-1-AP; 1:1,000; ProteinTech Group, Inc.), anti-Beclin-1 (cat. no. 11306-1-AP; 1:1,000; ProteinTech Group, Inc.), anti-autophagy-related 5 (ATG-5, cat. no. 66744-1-Ig; 1:1,000; ProteinTech Group, Inc.) and anti-AKT (cat. no. 60203-2-Ig; 1:1,000; ProteinTech Group, Inc.) overnight at $4^{\circ} \mathrm{C}$. The PVDF membranes were washed five times with TBS containing $0.02 \%$ Tween-20 $(1,000 \mathrm{ml}$ TBS and $2 \mathrm{ml}$ Tween-20). Following the primary incubation membranes were incubated with secondary antibodies [HRP-conjugated Affinipure Goat Anti-Mouse IgG(H+L), cat. no. SA00001-1; 1:10,000; ProteinTech Group, Inc. and HRP-conjugated Affinipure Goat Anti-Rabbit IgG(H+L), cat. no. SA00001-2; 1:10,000; ProteinTech Group, Inc.] at room temperature for 1 h. ECL chemiluminescence kit (Thermo Fisher Scientific, Inc.) was used to visualize protein bands. $\beta$-actin was used as an internal control. The intensity of protein bands was analyzed using ImageJ (version 146; National Institutes of Health). 
Reverse transcription-quantitative PCR (RT-qPCR). Total RNA was extracted using the RNA Extraction Kit (Takara Biotechnology Co., Ltd.), and cDNA was synthesized using a High-Capacity cDNA Archive kit (Takara Biotechnology Co., Ltd.). The following temperature protocol was used for reverse transcription: $37^{\circ} \mathrm{C}$ for $15 \mathrm{~min}$ and $85^{\circ} \mathrm{C}$ for $5 \mathrm{sec}$. Subsequently, the acquired cDNA was subjected to qPCR using an ABI 7500 Fast Real-Time PCR System (Applied Biosystems; Thermo Fisher Scientific, Inc.) and a SYBR-Green PCR Kit (Takara Biotechnology Co. Ltd.). The following thermocycling conditions were used for qPCR: $95^{\circ} \mathrm{C}$ for $30 \mathrm{sec}$, followed by 40 cycles of $95^{\circ} \mathrm{C}$ for $5 \mathrm{sec}$ and $65^{\circ} \mathrm{C}$ for $30 \mathrm{sec}$. The primers were synthesized by the Shanghai Sango Biotechnology Co. Ltd., and primer sequences are presented in Table I. Relative expression levels was analyzed using the $2^{-\Delta \Delta \mathrm{Cq}}$ method and normalized to $\beta$-actin as an internal control (15).

Immunofluorescence staining. THP-1 cells were seeded at a density of $1 \times 10^{5}$ cells/well into 6 -well plates and treated with $100 \mathrm{ng} / \mathrm{ml} \mathrm{PMA} \mathrm{at} 37^{\circ} \mathrm{C}$ for $24 \mathrm{~h}$ to transform into adherent macrophages. Then the cells of different treatment groups were incubated with M.tb (MOI:35) and vitamin D3 $(5 \mu \mathrm{mol} / \mathrm{ml})$ at $37^{\circ} \mathrm{C}$ for $24 \mathrm{~h}$. After being processed according to different treatment groups, the cells were washed three times at $37^{\circ} \mathrm{C}$ with PBS or Hanks' balanced salt solution to remove the culture medium. Subsequently, $1 \mathrm{ml}$ Fluo-4 AM $(1 \mu \mathrm{M})$ fluorescent probe (Thermo Fisher Scientific, Inc.) was added to the cells and incubated for $40 \mathrm{~min}$ in the dark at $37^{\circ} \mathrm{C}$. The NucBlue ${ }^{\mathrm{TM}}$ Fixed Cell ReadyProbes ${ }^{\mathrm{TM}}$ Reagent (DAPI; $5 \mu \mathrm{M}$; Invitrogen; Thermo Fisher Scientific) was used to stain the nucleus. Finally, a confocal laser scanning microscope was used to detect the fluorescence of Fluo-4 to verify changes in intracellular $\mathrm{Ca}^{2+}$ concentration.

Statistical analysis. All experiments were repeated at least three times. All data were analyzed using the SPSS 13.0 software (SPSS, Inc.). Unpaired Student's t-tests were used to compare differences between two groups, whereas one-way ANOVA followed by Tukey's post hoc test was used to compare differences between more than two groups. Data are presented as the mean \pm standard deviation. $\mathrm{P}<0.05$ was considered to indicate a statistically significant difference.

\section{Results}

Vitamin D3 decreases the viability of THP-1 cells in a dose-and time-dependent manner. To investigate the effects of vitamin D3 on THP-1 cell viability, the THP-1 cells were either treated with different concentrations of vitamin D3 $(0,5,20,50$ or $100 \mu \mathrm{mol} / \mathrm{ml})$ for $24 \mathrm{~h}$ (Fig. 1A) or treated with vitamin D3 $(5 \mu \mathrm{mol} / \mathrm{ml})$ for $0,6,12,24$ and $36 \mathrm{~h}$ (Fig. 1B). The results demonstrated that the viability of THP-1 cells was significantly decreased by vitamin D3 in a dose- and time-dependent manner compared with the respective control group; as vitamin D3 was dissolved in DMSO, cells treated with a culture medium of $0.03 \%$ DMSO were used as the control. It was demonstrated that when the vitamin D3 concentration reached $5 \mu \mathrm{mol} / \mathrm{ml}$, cellular viability was statistically different from the control group; therefore, this concentration was chosen for subsequent experiments.
Table I. Sequences of primers used for reverse transcriptionquantitative PCR.

\begin{tabular}{ll}
\hline Gene & \multicolumn{1}{c}{ Primer sequence $\left(5^{\prime} \rightarrow 3^{\prime}\right)$} \\
\hline AMPK & F: TTGAAACCTGAAAATGTCCTGCT \\
& R: GGTGAGCCACAACTTGTTCTT \\
LC3 & F: AACATGAGCGAGTTGGTCAAG \\
& R: GCTCGTAGATGTCCGCGAT \\
$\beta$-actin & F: GCCAACCGCGAGAAGATGA \\
& R: CCATCACGATGCCAGTGGTA
\end{tabular}

AMPK, AMP-activated protein kinase; $\mathrm{F}$, forward; $\mathrm{R}$, reverse.

Effect of vitamin D3 on $\mathrm{Ca}^{2+}$ concentration in THP-1 cells infected with M.tb. Impaired $\mathrm{Ca}^{2+}$ homeostasis can lead to cellular dysfunction and autophagy (16). Whether $\mathrm{Ca}^{2+}$ homeostasis of THP-1 cells infected by M.tb is altered under vitamin D3 treatment, and whether vitamin D3 could induce autophagy by inhibiting $\mathrm{Ca}^{2+}$ concentration was therefore investigated. To examine the $\mathrm{Ca}^{2+}$ homeostasis of THP-1 cells infected with $M . t b$ following vitamin D3 treatment, the intracellular $\mathrm{Ca}^{2+}$ concentration was quantified using Fluo-4 AM-treated THP-1 cells. Compared with the control group, M.tb infection for $24 \mathrm{~h}$ induced an $26 \%$ increase in $\mathrm{Ca}^{2+}$ concentration (Fig. 2A). Compared with the M.tb group, it was demonstrated that the vitamin D $3+M . t b(5,10$ and $20 \mu \mathrm{mol} / \mathrm{ml}$ ) groups had a significant inhibitory effect on $\mathrm{Ca}^{2+}$ concentration. Immunofluorescence assay results also demonstrated that vitamin D3 treatment markedly attenuated $\mathrm{Ca}^{2+}$ concentration compared with the control and M.tb groups (Fig. 2B). Compared with the vitamin D3 group, the $\mathrm{Ca}^{2+}$ concentration in the vitamin D3 + M.tb group was markedly enhanced. These results suggested that vitamin D3 treatment inhibited $\mathrm{Ca}^{2+}$ concentration in THP-1 cells infected by the M.tb.

Effect of vitamin D3 on autophagy-related factors in THP-1 cells. Vitamin D3 exhibited a significant inhibitory effect on the cellular viability of THP-1, aforementioned. However, whether vitamin D3 induced autophagy in THP-1 had not been explored. To determine whether vitamin D3 regulated autophagy in THP-1 cells, the protein expression levels of autophagy-associated proteins, p62, LC3, Beclin-1, ATG-5 and AKT, were analyzed by western blotting (Fig. 3). Compared to the M.tb group, treatment with vitamin D3 significantly increased the protein expression levels of p62, LC3II/LC3I, Beclin-1 and ATG-5 under M.tb infection (Fig. 3B-E). However, treatment with vitamin D3 significantly suppressed the protein expression levels of AKT under M.tb infection compared with the control (Fig. 3F). Moreover, the increase in LC3 and AMP activated protein kinase (AMPK) mRNA expression levels in the vitamin D3 and vitamin D3 + M.tb groups was higher compared with the M.tb group (Fig. 3G and H). Western blotting and RT-qPCR analyses indicated that vitamin D3 may stimulate autophagy in THP-1 cells. Taken together, these results suggested that vitamin D3 may inhibit M.tb infection by increasing autophagy in THP-1 cells. 

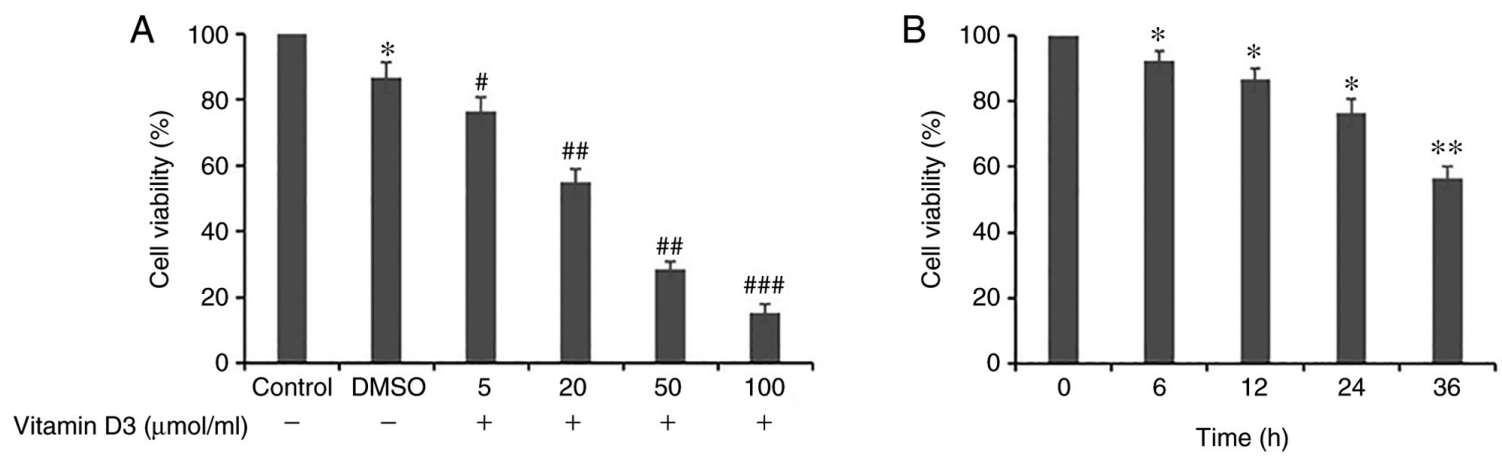

Figure 1. Effect of vitamin D3 on THP-1 cell viability. (A) THP-1 cells were treated with different concentrations of vitamin D3 $(0,5,20,50 \mathrm{and} 100 \mu \mathrm{mol} / \mathrm{ml})$ for $24 \mathrm{~h}$. (B) THP-1 cells were treated with vitamin D3 $(5 \mu \mathrm{mol} / \mathrm{ml})$ for various lengths of time $(0,6,12,24$ and $36 \mathrm{~h})$. The MTT assay was used to assess cellular viability. A microplate reader recorded the optical density change at $560 \mathrm{~nm}$. Data are presented as the mean \pm standard deviation of three independent experiments. ${ }^{*} \mathrm{P}<0.05,{ }^{* *} \mathrm{P}<0.01$ vs. control or $0 \mathrm{~h} ;{ }^{\#} \mathrm{P}<0.05,{ }^{\# \#} \mathrm{P}<0.01,{ }^{\# \#} \mathrm{P}<0.001$ vs. DMSO.
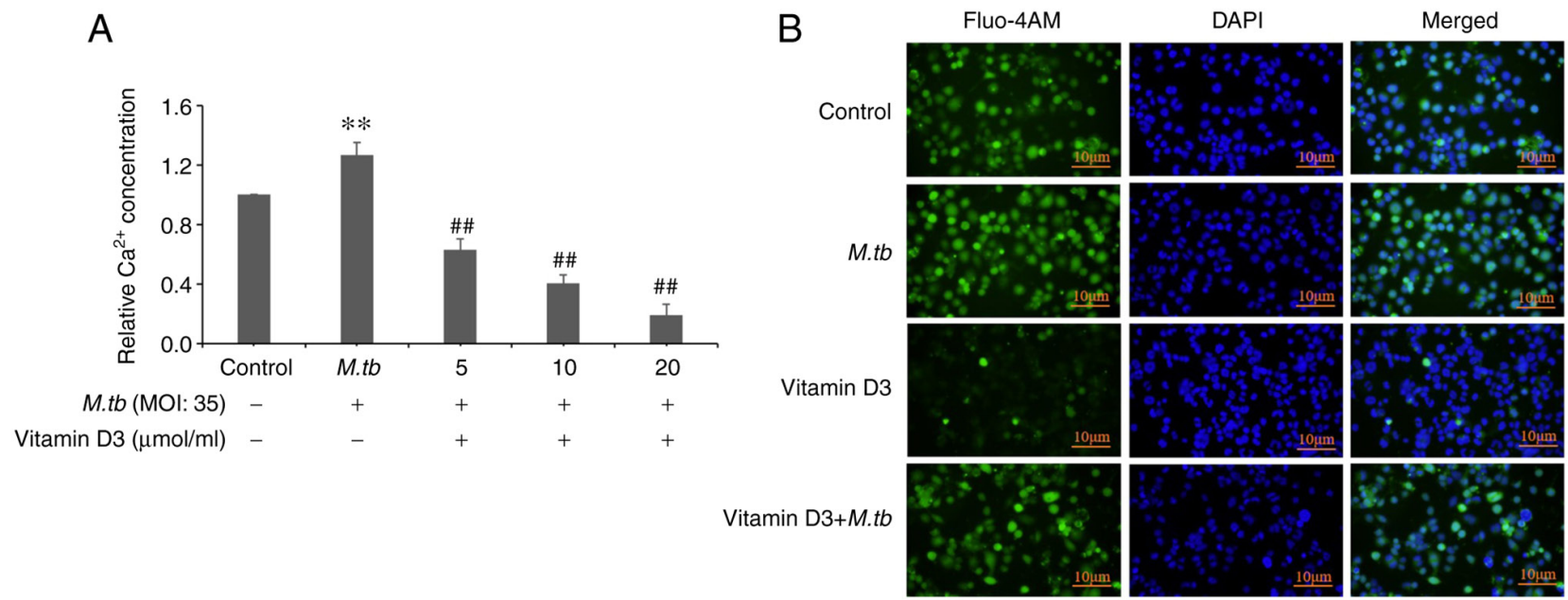

Figure 2. Vitamin D3 reduces $\mathrm{Ca}^{2+}$ concentration in THP-1 cells infected with M.tb. (A) Cells were treated with vitamin D3 (5, 10 and $\left.20 \mu \mathrm{mol} / \mathrm{ml}\right)$ and $/ \mathrm{or} M . t b$ for $24 \mathrm{~h}$ and then incubated with Fluo-4 AM $(1 \mu \mathrm{M})$ to determine $\mathrm{Ca}^{2+}$ concentration. (B) Cells were treated with vitamin D3 and/or M.tb for $24 \mathrm{~h}$ and then incubated with $1 \mathrm{ml}$ Fluo-4 AM $\left(1 \mu \mathrm{M}\right.$ ) fluorescent probe (green) and DAPI (blue) in the dark at $37^{\circ} \mathrm{C}$ for 40 min (scale bars, $10 \mu \mathrm{m}$ ). (A and B) Representative and relative changes in intracellular $\mathrm{Ca}^{2+}$ concentration following vitamin D3 treatment in THP-1 cells infected with M.tb. (compared with the control group). Data are presented as the mean \pm standard deviation of three independent experiments. ${ }^{* *} \mathrm{P}<0.01$ vs. control; and ${ }^{\# \#} \mathrm{P}<0.01$ vs. $M . t b$. AM, acetoxymethyl; M.tb, Mycobacterium tuberculosis.

Vitamin D3 attenuates lung damage following M.tb infection. After the Balb/c mice were treated intranasally with vitamin D3 and/or M.tb, pathological sections were produced and H\&E staining was performed. Pulmonary tissue sections from the control group (Fig. 4A) exhibited clear alveolar structure without edema, congestion or inflammatory cell infiltrates in the interstitial fluid. However, pulmonary tissues from the M.tb group (Fig. 4B) were damaged and inflammatory cell infiltrates and pathological changes were present. In the vitamin D3 (Fig. 4C) and vitamin $\mathrm{D} 3+M . t b$ groups (Fig. 4D), nasal administration was repeated for 7 days. Compared with the M.tb group, the degree of alveolar wall damage and infiltrating inflammatory cells in the vitamin D3 + M.tb group markedly decreased. These results suggested that vitamin D3 could reduce lung damage following M.tb infection by promoting cell autophagy.

\section{Discussion}

TB, caused by the bacterial pathogen M.tb, remains one of the deadliest infectious diseases, and the number of deaths and infections has continued to surge in the past 200 years (17). The latest WHO report estimates about 1.6 million global deaths annually from TB (18). Vitamin D3 induces anti-mycobacterial activity in mononuclear phagocytes (19) and an early study demonstrated that vitamin D3 is a potent antiproliferative agent in different tissues and cells, including the epithelial tissue, the vascular wall, the kidney, cancer cells and immune cells (20). Therefore, studying the autophagic mechanism of THP-1 cells infected with M.tb and induced by vitamin D3 will not only help understand the pathological mechanism of TB but also aid in discovering novel therapeutics for TB.

Previous study have indicated that THP-1 cells can be differentiated into mature macrophage-like cells using PMA (21). THP-1 cells resemble primary monocytes and macrophages in their morphological and functional properties and are a suitable, safe and reliable model to study macrophage functions and responses (22). THP-1 is an immortalized cell line with a high growth rate. Compared with PBMC-derived monocytes, THP-1 cells better facilitate reproducibility of findings. Furthermore, it has been reported that the THP-1 
A

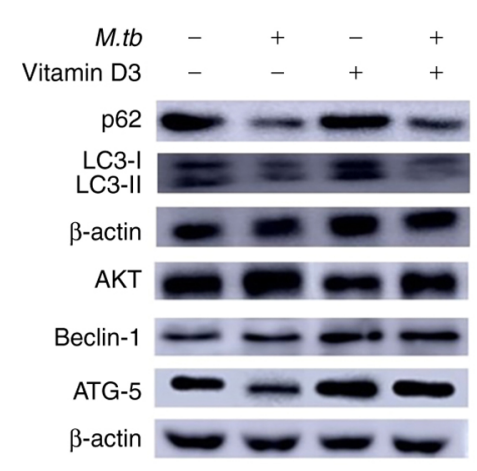

F

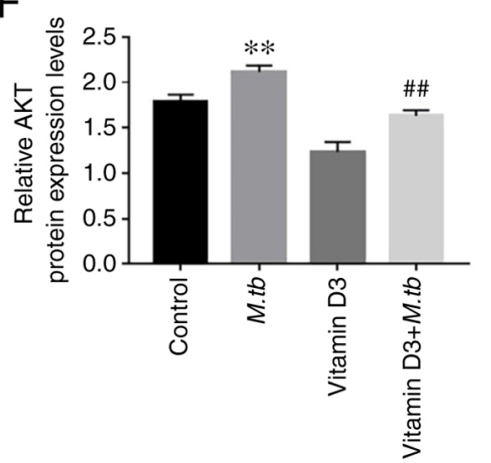

B

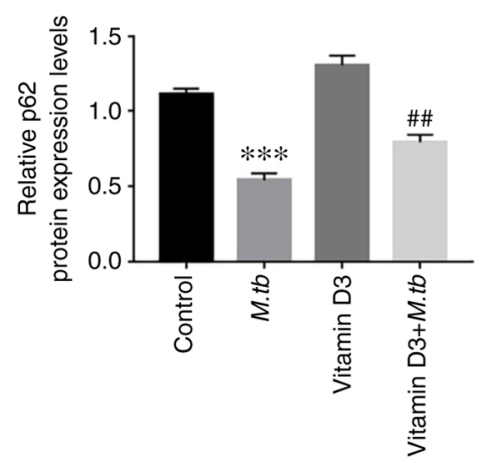

D

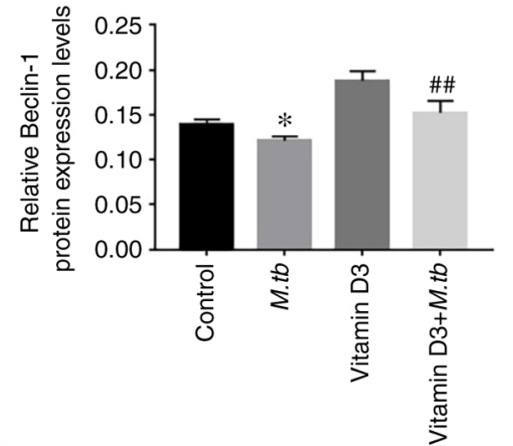

G

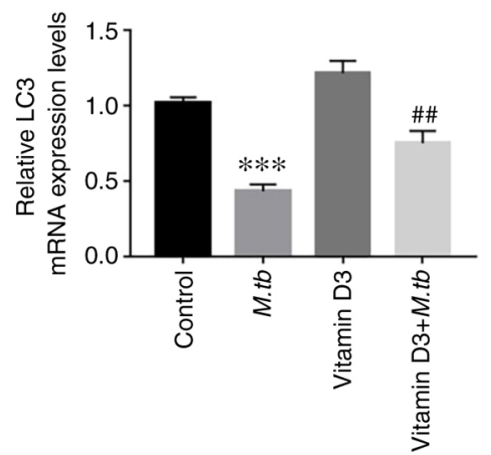

C

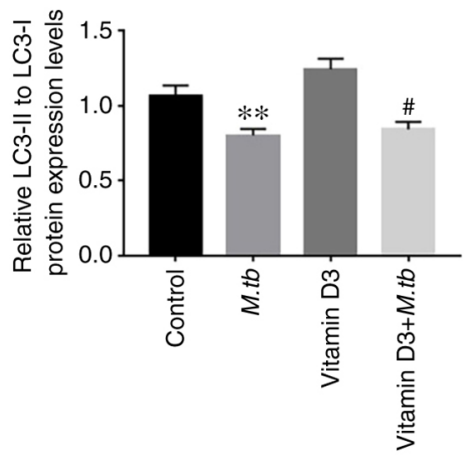

E

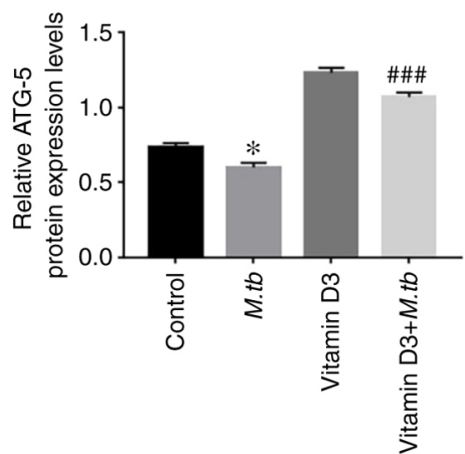

$\mathrm{H}$

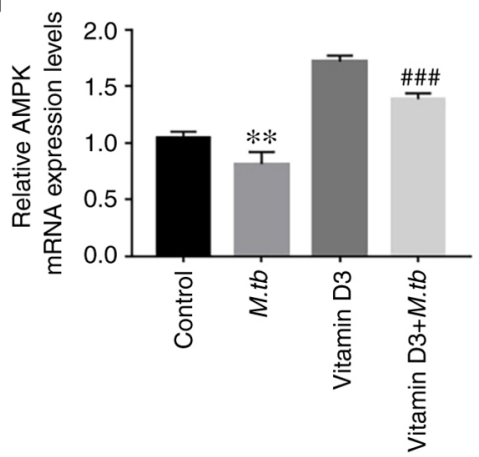

Figure 3. Effect of M.tb and vitamin D3 on THP-1 cell autophagy. (A) Following vitamin D3 and/or M.tb treatment for $24 \mathrm{~h}$, the protein expression levels of autophagy markers, including p62, LC3II/LC3I, Beclin-1, ATG-5 and AKT in THP-1 cells were analyzed by western blotting. (B) p62, (C) LC3II/LC3I, (D) Beclin-1, (E) ATG-5 and (F) AKT protein semi-quantitative expression levels. $\beta$-actin was used as a loading control. The mRNA expression levels of (G) LC3 and (H) AMPK were standardized using the $2^{-\triangle \Delta C q}$ method. Data are presented as the mean \pm standard deviation of three independent experiments. ${ }^{*} \mathrm{P}<0.05,{ }^{* *} \mathrm{P}<0.01,{ }^{* * *} \mathrm{P}<0.001$ vs. control; and ${ }^{\#} \mathrm{P}<0.05,{ }^{\# \#} \mathrm{P}<0.01,{ }^{\# \# \#} \mathrm{P}<0.001$ vs. M.tb. ATG-5, autophagy-related 5; M.tb, Mycobacterium tuberculosis; $\mathrm{p} 62$, sequestosome-1.

cell line is more sensitive to cellular $\mathrm{Ca}^{2+}$ signals. Therefore, human monocyte THP-1 cells were used in the present study to simulate vitamin D3-induced macrophage autophagy and to investigate the effective clearance of cells infected with M.tb by autophagy. In the present study, THP-1 cells were incubated with $M . t b$ for $24 \mathrm{~h}$ and the other treatment groups were treated with vitamin D3 or vitamin D3 + M.tb for $24 \mathrm{~h}$. The results demonstrated that incubation with $M . t b$ significantly decreased the protein expression levels of the autophagic proteins, p62, LC3II/LC3I, Beclin-1 and ATG-5. Compared with the M.tb group, the expression levels of p62, LC3II/LC3I, Beclin-1 and ATG-5 in the vitamin D3 + M.tb groups were significantly increased. Furthermore, the mRNA expression levels of LC3 and AMPK were also significantly increased in the vitamin D3 + M.tb group. Compared with the M.tb group, the autophagy-related protein, AKT, which is considered to be a negative regulator of autophagy, was reduced in the vitamin
$\mathrm{D} 3+$ M.tb groups. These results are consistent with another result of the present study, which demonstrated that vitamin D3 had a significant inhibitory effect on the $\mathrm{Ca}^{2+}$ concentration (23), which may have induced autophagy and explains the high efficiency of vitamin D3 in inhibiting the growth of cancer cells (24).

Autophagy serves a vital role in cell survival under certain stress conditions by scavenging proteins and damaged organelles to maintain cellular homeostasis and integrity (25). For example, the p62 protein is located at the autophagosome formation site and can bind to the autophagosomal localization protein LC3 and the family of ubiquitin-like proteins (26). ATG5, as autophagy proteins, is critical for autophagy at the stage of autophagosome formation (27). Moreover, Beclin-1 is a mammalian autophagic protein involved in diverse biological processes, including tumor suppression and cell death (28). AKT is also known as protein kinase $\mathrm{B}$, and it is an oncogenic 
A

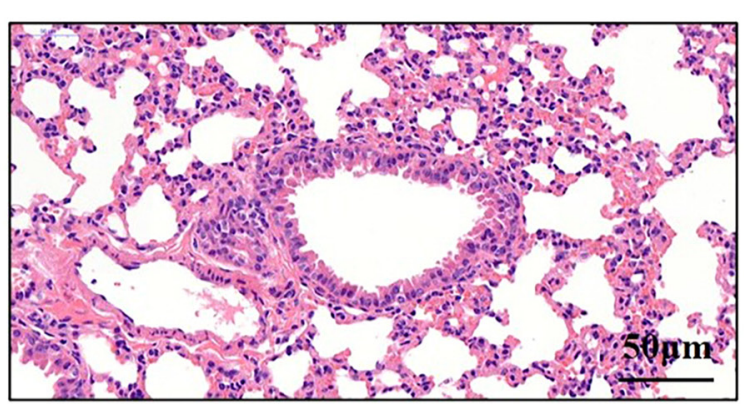

C

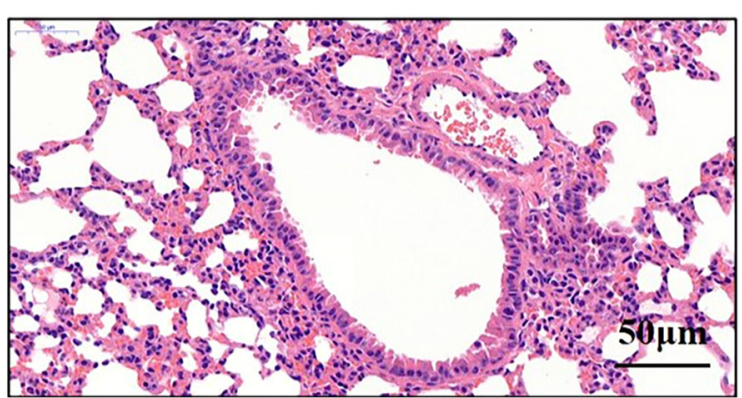

B
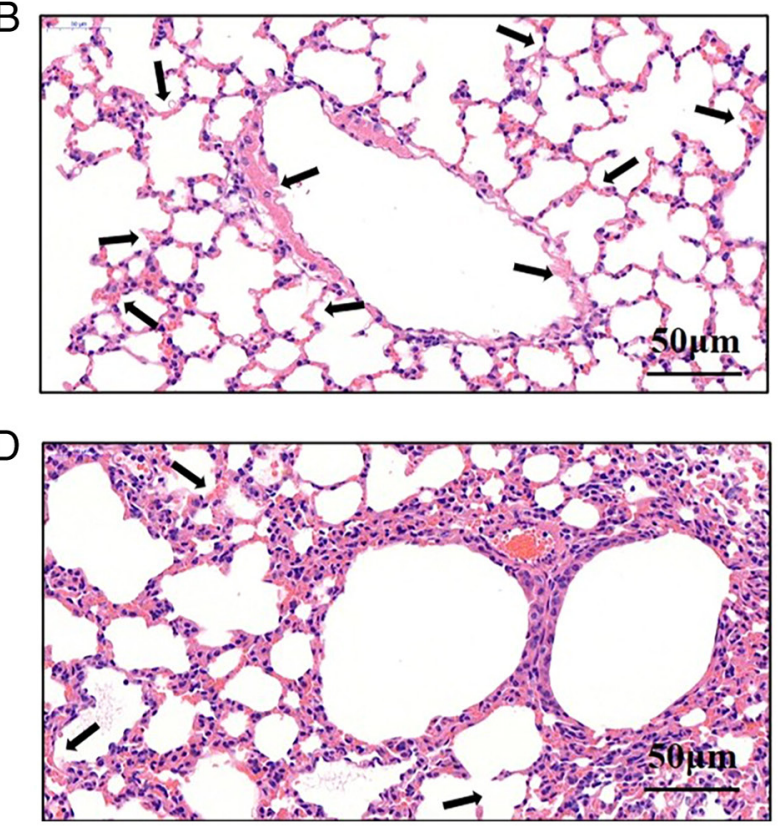

Figure 4. Vitamin D3 attenuates lung damage following M.tb infection. In Balb/c mice infected with M.tb, the morphological changes were observed in H\&E-stained pulmonary tissues. Representative photomicrographs illustrated characteristic lesions in 8-week-old Balb/c mice. Lavaged pulmonary tissues were fixed with $4 \%$ paraformaldehyde solution and were then treated conventionally for pathological sectioning and staining with H\&E. (A) Control group. (B) M.tb group. (C) Vitamin D3 group. (D) Vitamin D3 + M.tb group (arrows indicate amage to pulmonary tissues, inflammatory cell infiltration and pathological changes). Scale bar, $50 \mu \mathrm{M}$. M.tb, Mycobacterium tuberculosis.

protein that regulates cell survival, proliferation, growth and autophagy. As a negative regulator of autophagy and apoptosis, the AKT/mTOR signaling pathway serves an important role in regulating autophagy (29). AKT inhibitors directly inhibit the activity of AKT and therefore can attenuate cancer proliferation (30). LC3 and AMPK are essential cytokines involved in the regulation of autophagy (31). The data obtained in the present study demonstrated that vitamin D3 treatment resulted in a significant upregulation of p62, LC3II/LC3I, Beclin-1 and ATG-5 and a significant decrease in the AKT protein expression level. These results supported the hypothesis that vitamin D3 increases autophagy of THP-1 cells infected by M.tb. Therefore, vitamin D3 may stimulate innate immune antibacterial activity of THP-1 cells by enhancing mechanisms associated with autophagy.

A previous study has reported that reductions in the concentration of $\mathrm{Ca}^{2+}$ are associated with the triggering of autophagy (32). Furthermore, vitamin D3 alters $\mathrm{Ca}^{2+}$ homeostasis in cells (33). However, the effects of vitamin D3 in THP-1 cells following M.tb infection and its association with intracellular $\mathrm{Ca}^{2+}$ homeostasis, is still unknown. Therefore, in the present study the $\mathrm{Ca}^{2+}$ concentration in the cells treated with vitamin D3 was tracked by the cytoplasmic $\mathrm{Ca}^{2+}$ specific indicator Furo-4 AM. The results demonstrated that vitamin D3 significantly reduced $\mathrm{Ca}^{2+}$ concentration and significantly enhanced the expression levels of autophagy-associated proteins in THP-1 cells infected by $M . t b$ compared with the $M . t b$ group. These results therefore suggested that cytosolic $\mathrm{Ca}^{2+}$ may be crucial for vitamin D3-induced autophagy.

$M . t b$ is a typical parasitic bacterium that can evade the antimicrobial effect of macrophages through various mechanisms and survive in host immune cells, such as macrophages (34). A previous study reported that in $M . t b$-infected mice, the rupture of foam cells due to exacerbated infection and/or inflammation and the release of their contents likely sustains the disease pathology and generation of caseum, which leads to progressive destruction of lung tissues (35). Kimmey et al (36) demonstrated that $M . t b$ can evade autophagic responses in the mouse model. Induction of autophagy promotes maturation and acidification of M.tb phagosomes and their conversion into mycobactericidal organelles. The present study aimed to determine whether vitamin D3 could reduce lung damage following M.tb infection by promoting cell autophagy. Furthermore, various autophagy-related protein expression levels were analyzed to investigate this potential autophagic mechanism. The results of the present study suggested that incubation with M.tb significantly decreased autophagy-related protein expression levels, whereas vitamin D3 significantly promoted the activation of p62, LC3II/LC3I, Beclin-1 and ATG-5, and inhibited AKT protein expression levels, when induced by M.tb infection. Based on the above conclusions, it was speculated that vitamin D3 may promote phagosomal formation and autolysosomal maturation by significantly inhibiting the concentration of $\mathrm{Ca}^{2+}$ and likely enhancing the signal transduction in the host cell to promote the fusion of phagosomes and lysosomes. It is therefore possible that the autophagy signaling pathways of macrophages may have been activated to promote autophagy.

In conclusion, the present study demonstrated that the molecular mechanism of vitamin D3 promoted autophagy in THP-1 cells infected by M.tb. However, the precise mechanism by which vitamin D3 promotes the expression of autophagy-related proteins needs further research and it may also be necessary to repeat the experiments in a different type of macrophage cell line to further test the hypothesis. To the best of our knowledge, the present study is the first to report 
that vitamin D3 significantly reversed pulmonary injury of $M . t b$-infected mice, possibly via the autophagic pathway, thereby improving autophagic dysfunction. The results indicated that the mechanism of action of vitamin D3 may involve the attenuation of autophagic flux dysfunction through inhibition of the $\mathrm{Ca}^{2+}$ signaling pathway.

\section{Acknowledgements}

Not applicable.

\section{Funding}

The present study was funded by the National Natural Science Foundation of China (grant no. 31772710), Key Project of Research and Development of Ningxia Hui Autonomous Region of China (grant no. 2020BEG03019), Natural Science Foundation of Ningxia (grant no. 2021AAC03109), Key Project of Research and Development of Ningxia Hui Autonomous Region of China (grant no. 2019BBF02005) and Natural Science Foundation of Ningxia (grant no. 2021AAC03299).

\section{Availability of data and materials}

The datasets used and/or analyzed during the current study are available from the corresponding author on reasonable request.

\section{Authors' contributions}

YuW designed the project, revised the article and provided technical guidance. DX designed the project, revised the article and coordinated all aspects of the present work. YiW participated in all experiments, performed data analysis, created the figures and wrote the article. XL and FS were responsible for sample preparation and documentation. YiW and XL confirm the authenticity of all the raw data. All authors read and approved the final manuscript.

\section{Ethics approval and consent to participate}

All animal experiments and experimental protocols were approved by the Ethics Committee of Ningxia University (Yinchuan, China; approval no. 2020-013).

\section{Patient consent for publication}

Not applicable.

\section{Competing interests}

The authors declare that they have no competing interests.

\section{References}

1. Sharma $\mathrm{S}$ and Meena LS: Potential of $\mathrm{Ca}^{2+}$ in Mycobacterium tuberculosis $\mathrm{H}_{37} \mathrm{Rv}$ pathogenesis and survival. Appl Biochem Biotechnol 181: 762-771, 2017.

2. Chakaya JM, Harries AD and Marks GB: Ending tuberculosis by 2030-Pipe dream or reality? Int J Infect Dis 92S: S51-S54, 2020

3. de Martino M, Lodi L, Galli L and Chiappini E: Immune response to Mycobacterium tuberculosis: A narrative review. Front Pediatr 7: 350, 2019.
4. Xu G, Wang J, Gao GF and Liu CH: Insights into battles between Mycobacterium tuberculosis and macrophages. Protein Cell 5: 728-736, 2014.

5. Paik S and Jo EK: An interplay between autophagy and immunometabolism for host defense against mycobacterial infection. Front Immunol 11: 603951, 2020

6. Monteith GR, Davis FM and Roberts-Thomson SJ: Calcium channels and pumps in cancer: Changes and consequences. J Biol Chem 287: 31666-31673, 2012.

7. Huang W, Lu C, Wu Y, Ouyang S and Chen Y: T-type calcium channel antagonists, mibefradil and NNC-55-0396 inhibit cell proliferation and induce cell apoptosis in leukemia cell lines. J Exp Clin Cancer Res 34: 54, 2015.

8. Abu El Maaty MA and Wölfl S: Vitamin D as a novel regulator of tumor metabolism: Insights on potential mechanisms and implications for anti-cancer therapy. Int J Mol Sci 18: 3184, 2017.

9. Mondul AM, Weinstein SJ, Layne TM and Albanes D: Vitamin $\mathrm{D}$ and cancer risk and mortality: State of the science, gaps, and challenges. Epidemiol Rev 39: 28-48, 2017.

10. Martineau AR: Old wine in new bottles: Vitamin D in the treatment and prevention of tuberculosis. Proc Nutr Soc 71: 84-89, 2012.

11. Zhang L, Jiang X, Pfau D, Ling Y and Nathan CF: Type I interferon signaling mediates Mycobacterium tuberculosis-induced macrophage death. J Exp Med 218: e20200887, 2021.

12. Rizzuto R, Pinton P, Ferrari D, Chami M, Szabadkai G, Magalhães PJ, Di Virgilio F and Pozzan T: Calcium and apoptosis: Facts and hypotheses. Oncogene 22: 8619-8627, 2003.

13. Komohara Y, Fujiwara Y, Ohnishi $K$ and Takeya $M$ Tumor-associated macrophages: Potential therapeutic targets for anti-cancer therapy. Adv Drug Deliv Rev 99: 180-185, 2016.

14. Chanput W, Mes J, Vreeburg RA, Savelkoul HF and Wichers HJ: Transcription profiles of LPS-stimulated THP-1 monocytes and macrophages: A tool to study inflammation modulating effects of food-derived compounds. Food Funct 1: 254-261, 2010.

15. Kang P, Zhang W, Chen X, Yi X, Song P, Chang Y, Zhang S, Gao T, Li C and Li S: TRPM2 mediates mitochondria-dependent apoptosis of melanocytes under oxidative stress. Free Radic Biol Med 126: 259-268, 2018.

16. Wang $\mathrm{CH}$, Kao $\mathrm{CH}$, Chen YF, Wei YH and Tsai TF: Cisd2 mediates lifespan: Is there an interconnection among $\mathrm{Ca}^{2+}$ homeostasis, autophagy, and lifespan? Free Radic Res 48: 1109-1114, 2014.

17. Bussi C and Gutierrez MG: Mycobacterium tuberculosis infection of host cells in space and time. FEMS Microbiol Rev 43: 341-361, 2019.

18. Adeniji AA, Knoll KE and Loots DT: Potential anti-TB investigational compounds and drugs with repurposing potential in TB therapy: A conspectus. Appl Microbiol Biotechnol 104: 5633-5662, 2020.

19. Gough ME, Graviss EA and May EE: The dynamic immunomodulatory effects of vitamin D3 during Mycobacterium infection. Innate Immun 23: 506-523, 2017.

20. Svensson D, Nebel D and Nilsson BO: Vitamin D3 modulates the innate immune response through regulation of the hCAP-18/LL-37 gene expression and cytokine production. Inflamm Res 65: 25-32, 2016.

21. Riendeau CJ and Kornfeld H: THP-1 cell apoptosis in response to Mycobacterial infection. Infect Immun 71: 254-259, 2003.

22. Chanput W, Mes JJ and Wichers HJ: THP-1 cell line: An in vitro cell model for immune modulation approach. Int Immunopharmacol 23: 37-45, 2014.

23. Hashemipour S, Lalooha F, Zahir Mirdamadi S, Ziaee A and Dabaghi Ghaleh T: Effect of vitamin D administration in vitamin D-deficient pregnant women on maternal and neonatal serum calcium and vitamin D concentrations: A randomised clinical trial. Br J Nutr 110: 1611-1616, 2013.

24. Luong KV and Nguyen LT: The beneficial role of vitamin D and its analogs in cancer treatment and prevention. Crit Rev Oncol Hematol 73: 192-201, 2010.

25. Galati S, Boni C, Gerra MC, Lazzaretti M and Buschini A: Autophagy: A player in response to oxidative stress and DNA damage. Oxid Med Cell Longev 2019: 5692958, 2019.

26. Lamark T, Svenning S and Johansen T: Regulation of selective autophagy: The p62/SQSTM1 paradigm. Essays Biochem 61: 609-624, 2017.

27. Zheng W, Xie W, Yin D, Luo R, Liu M and Guo F: ATG5 and ATG7 induced autophagy interplays with UPR via PERK signaling. Cell Commun Signal 17: 42, 2019.

28. He $\mathrm{C}$ and Levine B: The beclin 1 interactome. Curr Opin Cell Biol 22: 140-149, 2010. 
29. Song M, Bode AM, Dong $\mathrm{Z}$ and Lee MH: AKT as a therapeutic target for cancer. Cancer Res 79: 1019-1031, 2019.

30. Revathidevi S and Munirajan AK: Akt in cancer: Mediator and more. Semin Cancer Biol 59: 80-91, 2019.

31. Revathidevi S and Munirajan AK: An overview of autophagy: Morphology, mechanism, and regulation. Antioxid Redox Signal 20: 460-473, 2014.

32. Kania E, Pająk B and Orzechowski A: Calcium homeostasis and ER stress in control of autophagy in cancer cells. Biomed Res Int 2015: 352794, 2015.

33. Pinton P, Giorgi C, Siviero R, Zecchini E and Rizzuto R: Calcium and apoptosis: ER-mitochondria $\mathrm{Ca}^{2+}$ transfer in the control of apoptosis. Oncogene 27: 6407-6418, 2008.

34. Padhi A, Pattnaik K, Biswas M, Jagadeb M, Behera A and Sonawane A: Mycobacterium tuberculosis LprE suppresses TLR2-dependent cathelicidin and autophagy expression to enhance bacterial survival in macrophages. J Immunol 203 2665-2678, 2019.
35. El-Sharkawy A and Malki A: Vitamin D signaling in inflammation and cancer: Molecular mechanisms and therapeutic implications. Molecules 25: 3219, 2020.

36. Kimmey JM, Huynh JP, Weiss LA, Park S, Kambal A, Debnath J, Virgin HW and Stallings CL: Unique role for ATG5 in neutrophil-mediated immunopathology during $M$. tuberculosis infection. Nature 528: 565-569, 2015.

c) (i) $\ominus$ This work is licensed under a Creative Commons cc) Attribution-NonCommercial-NoDerivatives 4.0 International (CC BY-NC-ND 4.0) License. 